\title{
On the Relationship between Two Notions of Compatibility for Bi-Hamiltonian Systems*
}

\author{
Manuele SANTOPRETE
}

Department of Mathematics, Wilfrid Laurier University, Waterloo, ON, Canada

E-mail:msantoprete@wlu.ca

Received June 30, 2015, in final form November 03, 2015; Published online November 07, 2015

http://dx.doi.org/10.3842/SIGMA.2015.089

\begin{abstract}
Bi-Hamiltonian structures are of great importance in the theory of integrable Hamiltonian systems. The notion of compatibility of symplectic structures is a key aspect of bi-Hamiltonian systems. Because of this, a few different notions of compatibility have been introduced. In this paper we show that, under some additional assumptions, compatibility in the sense of Magri implies a notion of compatibility due to Fassò and Ratiu, that we dub bi-affine compatibility. We present two proofs of this fact. The first one uses the uniqueness of the connection parallelizing all the Hamiltonian vector fields tangent to the leaves of a Lagrangian foliation. The second proof uses Darboux-Nijenhuis coordinates and symplectic connections.
\end{abstract}

Key words: bi-Hamiltonian systems; Lagrangian foliation; bott connection; symplectic connections

2010 Mathematics Subject Classification: 70H06; 70G45; 37K10

\section{Introduction}

Let $M$ be a smooth manifold of dimension $2 n$, and let $X$ be a vector field on $M$. Suppose that $X$ is Hamiltonian with respect to two different symplectic structures $\omega_{1}$ and $\omega_{2}$, that is,

$\mathbf{i}_{X} \omega_{1}=\mathbf{d} H \quad$ and $\quad \mathbf{i}_{X} \omega_{2}=\mathbf{d} K$,

where $H$ and $K$ are two, possibly distinct, Hamiltonian functions. Let us introduce the so called recursion operator $N=\omega_{2}^{\sharp} \omega_{1}^{b}: T M \rightarrow T M$, where $\omega^{b}: T M \rightarrow T^{*} M$ denotes the "musical" isomorphism induced by the symplectic form $\omega$, and $\omega^{\sharp}$ is its inverse. Then, as a consequence of the fact that $X$ is Hamiltonian with respect to two symplectic forms, the flow associated to $X$ preserves the eigenvalues of the recursion operator. Hence, if $N$ has $n$ functionally independent eigenvalues in involution, then, it is completely integrable via the Liouville-Arnold theorem.

A natural approach to integrability is to try to find sufficient conditions for the eigenvalues of the recursion operator to be in involution. Several sufficient conditions of this type have been found.

One such condition is based on the pioneering work of Magri [10] in the infinite-dimensional case. Magri and Morosi [12] showed that, if the sum of the Poisson tensor associated to $\omega_{1}$ and the one associated to $\omega_{2}$ is still a Poisson tensor, then the eigenvalues of the recursion operator are in involution. A similar claim is also present in the work of Gel'fand and Dorfman [8]. In this case we say that $\omega_{1}$ and $\omega_{2}$ are Magri-compatible, the triple $\left(M, \omega_{1}, \omega_{2}\right)$ is a bi-Hamiltonian manifold, and the quadruple $\left(M, \omega_{1}, \omega_{2}, X\right)$ is a bi-Hamiltonian system in Magri's sense if there exist functions $H_{1}$ and $H_{2}$ such that $X=\omega_{1}^{\sharp} \cdot \mathbf{d} H_{1}=\omega_{2}^{\sharp} \cdot \mathbf{d} H_{2}$. However, it is known that not all completely integrable Hamiltonian system are bi-Hamiltonian in Magri's sense. In fact

\footnotetext{
${ }^{\star}$ This paper is a contribution to the Special Issue on Analytical Mechanics and Differential Geometry in honour of Sergio Benenti. The full collection is available at http://www.emis.de/journals/SIGMA/Benenti.html
} 
there are results by Brouzet [3] and Fernandes [6] indicating that there are completely integrable Hamiltonian system that are not bi-Hamiltonian in Magri's sense. This limitation of Magri's definition stimulated the search for different notions of compatibility.

Bogoyavlenskij has given a sufficient condition that he calls strong dynamical compatibili$t y$ [2]. This condition requires the existence of a vector field $X$ that is Hamiltonian with respect to two symplectic structures $\omega_{1}$ and $\omega_{2}$, is completely integrable with respect to $\omega_{1}$, and it is non-degenerate in the following sense. The orbits of $X$ lie on Lagrangian tori and, in any local system of $\omega_{1}$-action-angle coordinates $(a, \alpha)$, the Hamiltonian function $H_{1}$ of $X$ associated to $\omega_{1}$ satisfies the following equation

$$
\operatorname{det}\left(\frac{\partial^{2} H_{1}}{\partial a_{i} \partial a_{j}}\right)(a) \neq 0
$$

A third notion of compatibility, was introduced by Fassò and Ratiu (see [5]) in order to study superintegrable systems, that is, systems with more than $n$ independent integrals of motion, and with motions on isotropic tori of dimension less than $n$, rather than on Lagrangian tori of dimension $n$. Let $\omega_{1}$ and $\omega_{2}$ be two symplectic forms on $M$. We say that a fibration (foliation) is bi-Lagrangian if the fibers (leaves) are Lagrangian with respect to both symplectic forms. Suppose there exist a bi-Lagrangian fibration of $M$. We say that $\omega_{1}$ and $\omega_{2}$ are bi-affinely compatible if the Bott connection (see Section 2 for a definition) associated to $\omega_{1}$ and the one associated to $\omega_{2}$ coincide.

Later we will explain the notion of Magri compatibility and bi-affine compatibility in more detail. In [5] Fassò and Ratiu wrote:

"It is not known to us whether our definition (as well as Bogoyavlenskij's) is more general than Magri's. If $\omega_{1}$ and $\omega_{2}$ are Magri compatible, then the eigenvalues of the recursion operator (if independent) define a bi-Lagrangian foliation. However, even when this foliation is a fibration and has compact and connected fibers, it is not clear whether, using the terminology of Definition 2, it is bi-affine."

This paper will be devoted to showing that Magri compatibility implies bi-affine compatibility in the simple case where the recursion operator has the maximal number of distinct eigenvalues. Note, however, that the converse is not true, since, as shown in [2] and [5], there exist bi-affinely compatible structures that are not compatible in Magri's sense. We believe it should be possible to tackle the general case by using Turiel's classification of Magri-compatible bi-Hamiltonian structures (see $[13,15])$.

We are aware of three different ways of proving that Magri compatibility implies bi-affine compatibility. The first proof uses the property that the Bott connection is the unique connection parallelizing all the Hamiltonian vector fields tangent to the leaves of a Lagrangian foliation. The second proof employs Darboux-Nijenhuis coordinates and the fact (proved in Proposition 5) that the restriction of a torsion-free symplectic connection to an involutive Lagrangian distribution coincides with the Bott connection in $L$. A third proof can be obtained directly by using the definition of Bott's connection given in equation (1). In this paper we will present only the first two proofs.

The first proof is more direct and has the advantage of avoiding the introduction of DarbouxNijenhuis coordinates and symplectic connections. The second proof, on the other hand, shows that Magri's compatibility condition allows to construct explicitly, in Darboux-Nijenhuis coordinates, two symplectic connections that have a special form.

Recall that, while Magri compatibility implies bi-affine compatibility, the converse, as mentioned above, does not hold. Since the restriction of a symplectic connection to an integrable Lagrangian distribution $L$ is the Bott connection in $L$ (as shown in Proposition 5) and bi-affine compatibility, by definition, concerns itself only with the Bott connection, it seems clear that 
that the difference between the two types of compatibility lies in the restrictions Magri's condition imposes on the allowable symplectic connections. Therefore, in our opinion, the link between symplectic connections and the notion of compatibility for bi-Hamiltonain systems deserves further investigation.

\section{Partial and symplectic connections}

In this section we briefly review some facts about partial connections and symplectic connections. We refer the reader to [7] for further details.

\section{$2.1 \quad$ Bott connection}

We first recall the definition of a partial connection.

Definition 1. Let $M$ be a manifold, $V$ a vector bundle over $M, L$ a distribution on $M$. Let $\Gamma(V)$ denote the space of sections of $V$, and $\Gamma(L)$ the space of vector fields tangent to $L$. A partial linear connection in $V$ along $L$ is a bilinear map

$$
\nabla: \Gamma(L) \times \Gamma(V) \rightarrow \Gamma(V):(X, Y) \rightarrow \nabla_{X} Y,
$$

such that

1) $\nabla_{f X} Y=f \nabla_{X} Y$ (i.e., $\nabla$ is linear in $X$ ),

2) $\nabla_{X}(f Y)=f \nabla_{X} Y+(X[f]) Y$ (i.e., $\nabla$ is a derivation),

for $X \in \Gamma(L), f \in C^{\infty}(M)$, and $Y \in \Gamma(V)$.

Suppose $L$ is an involutive distribution on $M$, and let $L^{\perp}$ be the annihilator of $L$, that is, the vector subbundle of $T^{*} M$ consisting of 1 -forms that vanish on $L$. The partial linear connection $\tilde{\nabla}^{B}$ in $L^{\perp}$ along $L$ defined by

$$
\tilde{\nabla}_{X}^{B} \alpha=\mathcal{L}_{X} \alpha, \quad \text { for } \quad X \in \Gamma(L), \quad \alpha \in \Gamma\left(L^{\perp}\right)
$$

is the Bott connection associated with the distribution $L$.

Now assume that $(M, \omega)$ is an almost-symplectic manifold (that is, $\omega$ is a non-degenerate 2form), and $L$ is an involutive Lagrangian distribution with respect to $\omega$. By Frobenius theorem $L$ is also integrable, that is, each point of $M$ is contained in an integral manifold of $L$. Moreover, the collection of all maximal connected integral manifolds of $L$ forms a foliation of $M$ (see [9] for more details). Since the "musical" isomorphism $\omega^{b}: T M \rightarrow T^{*} M$ and its inverse $\omega^{\sharp}: T^{*} M \rightarrow T M$ restrict to $\omega^{b}: \Gamma(L) \rightarrow \Gamma\left(L^{\perp}\right)$ and $\omega^{\sharp}: \Gamma\left(L^{\perp}\right) \rightarrow \Gamma(L)$ we can use them to define a partial linear connection in $L$ along $L$.

Definition 2. Let $L$ be an involutive Lagrangian distribution on the almost symplectic manifold $(M, \omega)$. The partial connection

$$
\nabla^{B}: \Gamma(L) \times \Gamma(L) \rightarrow \Gamma(L):(X, Y) \rightarrow \nabla_{X} Y
$$

defined by

$$
\nabla_{X}^{B} Y=\omega^{\sharp} \mathcal{L}_{X}\left(\omega^{b} Y\right)
$$

is called, by an abuse of terminology, the Bott connection in $L$. 
The Bott connection can also be defined with the following formula:

$$
\omega\left(\nabla_{X}^{B} Y, Z\right)=X[\omega(Y, Z)]-\omega(Y,[X, Z])
$$

for $X, Y \in \Gamma(L), Z \in \Gamma(T M)$.

It can be shown that $\nabla^{B}$ defines a flat partial connection. See [7] for more details. However, in general, the Bott connection is not torsion-free, in fact we have the following proposition.

Proposition 1. Let $(M, \omega)$ be an almost symplectic manifold, and let $L$ be an involutive Lagrangian distribution on $M$. Then, if $\omega$ is closed, the Bott connection $\nabla^{B}$ in $L$ has zero torsion. More generally, the torsion tensor $T^{B}$ of $\nabla^{B}$, defined by

$$
T^{B}(X, Y)=\nabla_{X}^{B} Y-\nabla_{Y}^{B} X-[X, Y]
$$

is related to the exterior derivative of $\omega$ by the formula

$$
\mathbf{d} \omega(X, Y, Z)=\omega\left(T^{B}(X, Y), Z\right) \quad \text { for } \quad X, Y \in \Gamma(L), \quad Z \in \Gamma(T M) .
$$

A proof of this statement can be found in [7].

Another important property of the Bott connection is that it is the unique connection parallelizing all the Hamiltonian vector fields tangent to the leaves of a Lagrangian foliation. More precisely we have the following theorem.

Theorem 1. Suppose $(M, \omega)$ is a $2 n$-dimensional symplectic manifold, and $U$ an open subset of $M$. Let $f_{1}, \ldots, f_{n}$ be a set of smooth functions defined on $U$ such that

1) the $f_{i}$ are pairwise in involution, that is, $\left\{f_{i}, f_{j}\right\}=0$,

2) the differentials are linearly independent, that is, $\mathbf{d} f_{i} \wedge \cdots \wedge \mathbf{d} f_{n} \neq 0$.

Then the $f_{i}$ define a Lagrangian foliation of $U$, with leaves of the form

$$
N^{c}=\left\{x \mid f_{1}(x)=c_{1}, \ldots, f_{n}(x)=c_{n}\right\},
$$

and the Bott connection

$$
\nabla_{X}^{B} Y=\omega^{\sharp} \mathcal{L}_{X}\left(\omega^{b} Y\right)
$$

is the unique connection parallelizing all the Hamiltonian vector fields tangent to the leaves.

Proof. The fact that the $f_{i}$ define a Lagrangian foliation is well known. Let $Y=\omega^{\sharp} \cdot \mathbf{d} g$ be a Hamiltonian vector field tangent to the leaves of the foliation. We prove that the Bott connection parallelizes the Hamiltonian vector fields tangent to the leaves. Clearly, we have

$$
\begin{aligned}
\nabla_{X_{i}}^{B} Y & =\omega^{\sharp} \mathcal{L}_{X_{i}}\left(\omega^{b} Y\right)=\omega^{\sharp} \mathcal{L}_{X_{i}}(\mathbf{d} g) \stackrel{\text { (by Cartan's formula) }}{=} \omega^{\sharp}\left(\mathbf{i}_{X_{i}} \mathbf{d}^{2} g+\mathbf{d}\left(\mathbf{i}_{X_{i}} \mathbf{d} g\right)\right) \\
& =\omega^{\sharp}\left(\mathbf{d}\left(\mathbf{i}_{X_{i}} \mathbf{d} g\right)\right)=\omega^{\sharp}\left(\mathbf{d}\left\langle\mathbf{d} g, X_{i}\right\rangle\right)=\omega^{\sharp}\left(\mathbf{d}\left\langle\omega^{b} Y, X_{i}\right\rangle\right)=\omega^{\sharp}\left(\mathbf{d}\left(\omega\left(Y, X_{i}\right)\right)\right)=0,
\end{aligned}
$$

since $\omega\left(Y, X_{i}\right)=0$. This is because $Y$ is tangent to the leaves and the leaves are Lagrangian submanifolds. Now, suppose $X=\sum_{i=1}^{n} a_{i} X_{i}$, then

$$
\nabla_{X} Y=\sum a_{i} \nabla_{X_{i}}^{B} Y=0
$$

Hence, $\nabla^{B}$ parallelizes all the Hamiltonian vector fields $Y=\omega^{\sharp} \cdot \mathbf{d} g$ tangent to the leaves. 
To show uniqueness suppose there is another connection $\left(\nabla^{B}\right)^{\prime}$ with the same property. Then, there is a tensor $S(X, Y)$ such that $\left(\nabla^{B}\right)_{X}^{\prime} Y=\nabla_{X}^{B} Y+S(X, Y)$. Hence, we have

$$
0=\left(\nabla^{B}\right)_{X_{i}}^{\prime} X_{j}=\nabla_{X_{i}}^{B} X_{j}+S\left(X_{i}, X_{j}\right)=S\left(X_{i}, X_{j}\right)
$$

Consequently, $S(X, Y)=0$ for all $X, Y$. In fact, let $X=\sum_{i=1}^{n} a_{n} X_{i}$ and $Y=\sum_{i=1}^{n} b_{i} X_{i}$, then

$$
S(X, Y)=\sum_{i, j} a_{i} b_{j} S\left(X_{i}, X_{j}\right)=0
$$

by linearity, since $S\left(X_{i}, X_{j}\right)=0$.

Remark 1. Suppose the hypotheses of the theorem above are verified and let $Y_{1}, \ldots, Y_{n}$ be Hamiltonian vector fields that, at each point, span the tangent plane to the leaves of the foliation. Then, the Bott connection is the unique connection parallelizing all the vector fields $Y_{i}$. The proof of this is given by a computation similar to the one in the proof of the theorem above.

\subsection{Symplectic connections}

Definition 3. Let $(M, \omega)$ be an almost symplectic manifold. A symplectic connection on $(M, \omega)$ is a bilinear map

$$
\nabla: \Gamma(T M) \times \Gamma(T M) \rightarrow \Gamma(T M):(X, Y) \rightarrow \nabla_{X} Y
$$

such that $\nabla$ is a linear connection, that is,

1) $\nabla_{f X}=f \nabla_{X} Y$

2) $\nabla_{X}(f Y)=f \nabla_{X} Y+(X[f]) Y$,

and parallelizes $\omega$, that is,

3) $\left(\nabla_{X} \omega\right)(Y, Z)=0$ for all $X, Y, Z \in \Gamma(T M)$, where $\nabla_{X} \omega$ denotes the covariant derivative of $\omega$, given by the formula

$$
\left(\nabla_{X} \omega\right)(Y, Z)=X[\omega(Y, Z)]-\omega\left(\nabla_{X} Y, Z\right)-\omega\left(Y, \nabla_{X} Z\right) .
$$

Note that here we adhere to the terminology of [7]. Other authors incorporate the requirement of being torsion-free, namely

$$
T(X, Y)=\nabla_{X} Y-\nabla_{Y} X-[X, Y]=0
$$

for all $X, Y \in \Gamma(T M)$, in the definition of a symplectic connection.

The existence of torsion-free symplectic connections is ensured, in the case of symplectic manifolds, by the following proposition.

Proposition 2. Let $(M, \omega)$ be a symplectic manifold. Then, there is a torsion-free symplectic connection on $M$.

See [1] for a proof. On the other hand, if the manifold is not symplectic (i.e., $\omega$ is not closed) a torsion-free symplectic connection does not exist, in fact we have the following proposition. 
Proposition 3. Let $(M, \omega)$ be an almost symplectic manifold, and let $\nabla$ be a symplectic connection on $M$. Then, the torsion tensor $T$ of $\nabla$ is related to the exterior derivative of $\omega$ by the formula

$$
\mathbf{d} \omega(X, Y, Z)=\omega(T(X, Y), Z)+\omega(T(Z, Y), X)+\omega(T(Z, X), Y) .
$$

Consequently, if there is a torsion-free symplectic connection $\nabla$ on $M, \omega$ must be closed.

Suppose $L$ is a distribution on $M$. Let $\Gamma(L)$ denote the set of all vector fields tangent to $L$, and let $\Gamma(T M)$ denote the set of all vector fields on $M$. Recall that a connection $\nabla$ is said to parallelize (or preserve) a distribution $L$ if $\nabla_{X} Y \in \Gamma(L)$ for all vector fields $X \in \Gamma(T M)$ and all vector fields in $Y \in \Gamma(L)$. The following result, proved in [7], links symplectic connections and Lagrangian distributions.

Proposition 4. Let $(M, \omega)$ be an almost symplectic manifold and let $L$ be a Lagrangian distribution on $M$. If there exists a torsion-free symplectic connection $\nabla$ on $M$ that preserves $L$, then $\omega$ must be closed (that is, symplectic), and $L$ must be involutive.

Finally, we state and prove a proposition, also proved in [7], that will be essential for the main result of this paper.

Proposition 5. Let $(M, \omega)$ be a symplectic manifold and let $L$ be an involutive Lagrangian distribution, then the restriction of any symplectic torsion-free connection $\nabla$ preserving $L$ to $L$ coincides with the Bott connection in L.

Proof. From the definition of symplectic connection:

$$
\omega\left(\nabla_{X} Y, Z\right)=X[\omega(Y, Z)]-\omega\left(Y, \nabla_{X} Z\right)=X[\omega(Y, Z)]-\omega\left(Y, \nabla_{Z} X\right)-\omega(Y,[X, Z]),
$$

where the second equality holds because, by hypothesis, the connection is torsion-free. Since $\nabla$ preserves $L$ we have that, if $X \in \Gamma(L)$, then $\nabla_{Z} X \in \Gamma(L)$. Furthermore, if $X, Y \in \Gamma(L)$, then, since the distribution is Lagrangian, we have that $\omega\left(Y, \nabla_{Z} X\right)=0$, and

$$
\omega\left(\nabla_{X} Y, Z\right)=X[\omega(Y, Z)]-\omega(Y,[X, Z])
$$

This last equation agrees with equation (2) for the Bott connection.

\section{Darboux-Nijenhuis coordinates}

Suppose $\omega_{1}$ and $\omega_{2}$ are Magri-compatible symplectic forms on a smooth $2 n$-dimensional manifold $M$, and let $N=\omega_{2}^{\sharp} \omega_{1}^{b}: T M \rightarrow T M$ be the recursion operator.

Magri's notion of compatibility can be equivalently expressed by saying that the Nijenhuis torsion of the recursion operator vanishes for all vector fields $X, Y$, that is,

$$
T_{N}(X, Y)=[N X, N Y]-N[N X, Y]-N[X, N Y]+N^{2}[X, Y]=0 .
$$

A proof of this fact can be found, for instance, in [11]. A tensor field with vanishing torsion is called a Nijenhuis tensor field. A Nijenhuis tensor field is compatible with a symplectic form $\omega$ if

1) $\omega^{b} N=N^{*} \omega^{b}$

2) $\mathbf{d} \omega(N X, Y, \cdot)-\mathbf{d} \omega(N Y, X, \cdot)+\mathbf{d} \Omega(Y, X, \cdot)=0$ for all $X, Y$, 
where $N^{*}$ is the adjoint tensor of $N$, and $\Omega$ is defined by the following expression

$$
\Omega(X, Y)=\left\langle\Omega^{b} X, Y\right\rangle=\left\langle\left(\omega^{b} N\right) X, Y\right\rangle \text {. }
$$

The first condition ensures $\Omega$ is skew symmetric, while the second ensures that $\Omega$ is closed.

A triple $(M, \omega, N)$, where $\omega$ is a symplectic form and $N$ is a compatible Nijenhuis tensor field, is called an $\omega N$ manifold. The definition of $\omega N$ manifold first appeared in the work of Magri and Morosi [12]. A manifold $M$ with two Magri-compatible symplectic forms is an important example of an $\omega N$ manifold.

If $\left(M, \omega_{1}, \omega_{2}, X\right)$ is a bi-Hamiltonian system (in Magri's sense) then it is possible to use the recursion operator to create a sequence of functions in involution that commute with the Hamiltonians $H_{1}$ and $H_{2}$. Then, if a sufficient number of integrals are functionally independent, the system is completely integrable. Such sequence of functions can be constructed by using the trace of powers of the recursion operator as follows

$$
I_{k}=\frac{1}{k} \operatorname{Tr}\left(N^{k}\right) .
$$

Since $T_{N}(X, Y)=0$ it can be shown that the differentials $\mathbf{d} I_{k}$ satisfy the Lenard recursion relation $N^{*} \mathbf{d} I_{k}=\mathbf{d} I_{k+1}$, or equivalently, since $N^{*}=\omega_{1}^{b} \omega_{2}^{\sharp}$, they satisfy $\omega_{2}^{\sharp} \cdot \mathbf{d} I_{k}=\omega_{1}^{\sharp} \cdot \mathbf{d} I_{k+1}$. Since the $\mathbf{d} I_{k}$ 's fulfill the recursion relation it is easy to show that $\left\{I_{i}, I_{j}\right\}_{1}=\left\{I_{i}, I_{j}\right\}_{2}=0$, where $\{,\}_{1}$ and $\{,\}_{2}$ are the Poisson brackets associated with $\omega_{1}$ and $\omega_{2}$, respectively. Thus, the $I_{j}$ are in involution. For a more detailed account of this construction we refer the reader to $[11,12]$. It is convenient to give the following definition (see for example [14]):

Definition 4. A point $p$ of an $\omega N$ manifold is a regular point if $N$ has the maximal number $n=$ $\frac{1}{2} \operatorname{dim}(M)$ of distinct, functionally independent, eigenvalues $\lambda_{1}, \lambda_{2}, \ldots, \lambda_{n}$ (i.e., the differentials $\mathbf{d} \lambda_{1}, \ldots, \mathbf{d} \lambda_{n}$ are linearly independent at $p$ ). An open set $U \subset M$ is called regular if each point of $U$ is a regular point.

We remark that, in the definition above, it is enough to require that the eigenvalues are distinct and non-constant, since the latter automatically implies their independence.

Furthermore, note that, in a regular open set $U$, since the $\lambda_{i}$ are functionally independent, it follows that the $I_{i}$ 's are also functionally independent (see, for instance, [4] for a proof), and so the $I_{i}$ define a bi-Lagrangian foliation, namely, a foliation Lagrangian with respect to $\omega_{1}$ and $\omega_{2}$. In this setting, it can also be shown that the eigenvalues are in involution $\left\{\lambda_{i}, \lambda_{j}\right\}_{1}=\left\{\lambda_{i}, \lambda_{j}\right\}_{2}=0$ (see $[4,11]$ ), and the $\lambda_{i}$ also define the same bi-Lagrangian foliation. With these definitions we have the following proposition.

Proposition 6. Let $\left(M, \omega_{1}, N\right)$ be an $\omega N$ manifold. Each regular point has an open neighborhood where there exist coordinates $(\boldsymbol{\lambda}, \boldsymbol{\mu})=\left(\lambda_{1}, \ldots, \lambda_{n}, \mu_{1}, \ldots, \mu_{n}\right)$ (where the $\lambda_{i}$ 's are the eigenvalues of $N)$, called Darboux-Nijenhuis coordinates, satisfying the following two properties:

1) $\omega_{1}=\sum_{i} \mathbf{d} \lambda_{i} \wedge \mathbf{d} \mu_{i}$, that is, they are Darboux-coordinates for $\omega_{1}$,

2) $N^{*} \mathbf{d} \lambda_{i}=\lambda_{i} \mathbf{d} \lambda_{i}$ and $N^{*} \mathbf{d} \mu_{i}=\lambda_{i} \mathbf{d} \mu_{i}$.

See [11] for a sketch of the proof of this statement.

In these coordinates the tensor $N$ takes the diagonal form

$$
N=\left[\begin{array}{cc}
\boldsymbol{\Lambda}_{n} & \mathbf{0}_{n} \\
\mathbf{0}_{n} & \boldsymbol{\Lambda}_{n}
\end{array}\right]
$$


where $\boldsymbol{\Lambda}_{n}=\operatorname{diag}\left(\lambda_{1}, \ldots, \lambda_{n}\right)$, and $\mathbf{0}_{n}$ is the $n \times n$ matrix with zero entries. Moreover $\omega_{2}^{\sharp}=$ $N\left(\omega_{1}^{b}\right)^{-1}$ takes the form

$$
\omega_{2}^{\sharp}=\left[\begin{array}{cc}
\mathbf{0}_{n} & \boldsymbol{\Lambda}_{n} \\
-\boldsymbol{\Lambda}_{n} & \mathbf{0}_{n}
\end{array}\right],
$$

and since the matrix of $\omega_{2}^{\sharp}$ is the inverse of that of $\omega_{2}^{b}$ and the matrix of $\omega_{2}^{b}$ is the negative of that of $\omega_{2}$ we have

$$
\omega_{2}=\left[\begin{array}{cc}
\mathbf{0}_{n} & \boldsymbol{\Lambda}_{n}^{-1} \\
-\boldsymbol{\Lambda}_{n}^{-1} & \mathbf{0}_{n}
\end{array}\right] .
$$

Remark 2. The definition of Darboux-Nijenhuis coordinates can be generalized to each set of Darboux coordinates in which $N$ takes the diagonal form. With such more general definition, one can manage the cases in which the eigenvalues are not independent.

\section{Bi-affine compatibility}

We now recall the definition of compatibility due to Fassò and Ratiu (see [5]) in the special case the fibration is not only bi-isotropic but also bi-Lagrangian, since this is the only relevant case for our purposes. We refer to this type of compatibility as bi-affine compatibility.

Definition 5. Let $M$ be a smooth manifold of dimension $2 n$ and let $\omega_{1}$ and $\omega_{2}$ be two symplectic structures on $M$. Assume there exists a bi-Lagrangian fibration $\pi$ of $M$ with compact connected fibers which is bi-affine (i.e., the restriction of the Bott connections $\nabla_{1}^{B}$ and $\nabla_{2}^{B}$ associated with $\omega_{1}$ and $\omega_{2}$ to the fibers, coincide). Then we say that $\omega_{1}$ and $\omega_{2}$ are bi-affinely compatible or $\pi$-compatible.

\section{Relationship between Magri's notion of compatibility and bi-affine compatibility}

Suppose we have a manifold $M$ with two Magri-compatible symplectic forms $\omega_{1}$ and $\omega_{2}$. If every point is regular, then, as we mentioned in Section 3, the $I_{k}$ 's (with $I_{k}=\frac{1}{k} \operatorname{Tr}\left(N^{k}\right)$ ) define a bi-Lagrangian foliation with associated distribution $L$. The link between Magri's notion of compatibility and bi-affine compatibility is given in the following theorem.

Theorem 2. Under the hypothesis above the Bott connections

$$
\left(\nabla_{B}^{1}\right)_{X} Y=\omega_{1}^{\sharp} \mathcal{L}_{X}\left(\omega_{1}^{b} Y\right) \quad \text { and } \quad\left(\nabla_{B}^{2}\right)_{X} Y=\omega_{2}^{\sharp} \mathcal{L}_{X}\left(\omega_{2}^{b} Y\right)
$$

in $L$ coincide. If, in particular, the Lagrangian foliation is a Lagrangian fibration with compact connected fibers then Magri's compatibility implies bi-affine compatibility.

Proof. Let $X_{k}=\omega_{1}^{\sharp} \cdot \mathbf{d} I_{k+1}$ for $k=1, \ldots, n$. Since $\omega_{1}$ and $\omega_{2}$ are Magri compatible, as we mentioned in Section 3, it follows that

$$
X_{k}=\omega_{1}^{\sharp} \cdot \mathbf{d} I_{k+1}=\omega_{2}^{\sharp} \cdot \mathbf{d} I_{k}, \quad k=1, \ldots, n .
$$

Hence, the $X_{k}$ 's are Hamiltonian with respect to both symplectic structures and, by Theorem 1 , these vector fields are parallel with respect to both $\nabla_{B}^{1}$ and $\nabla_{B}^{2}$. Since the $X_{k}$ 's span the tangent space to the leaf, by the uniqueness results of Theorem 1 and the following remark, it may be concluded that the connections $\nabla_{B}^{1}$ and $\nabla_{B}^{2}$ coincide. 
Under the hypothesis of the theorem above, as a consequence of Proposition 6, we have that, in a neighborhood of every point, there exist Darboux-Nijenhuis coordinates. As a consequence, we can give an alternative proof of the theorem above that employs symplectic connections and Darboux-Nijenhuis coordinates. This approach requires constructing explicitly the symplectic connections associated with $\omega_{1}$ and $\omega_{2}$. This will be done in the proof of the following theorem.

Theorem 3. Under the hypothesis above there are two torsion-free symplectic connections $\nabla^{1}$ and $\nabla^{2}$, symplectic with respect to $\omega_{1}$ and $\omega_{2}$, respectively, and such that

1) they preserve the Lagrangian distribution $L$ associated to the foliation defined by the eigenvalues $\lambda_{1}, \ldots, \lambda_{n}$,

2) the restrictions of $\nabla^{1}$ and $\nabla^{2}$ to $L$ coincide with the Bott connections defined by $\left(\nabla_{B}^{1}\right)_{X} Y=$ $\omega_{1}^{\sharp} \mathcal{L}_{X}\left(\omega_{1}^{b} Y\right)$ and $\left(\nabla_{B}^{2}\right)_{X} Y=\omega_{2}^{\sharp} \mathcal{L}_{X}\left(\omega_{2}^{b} Y\right)$, respectively,

3) the restrictions of $\nabla^{1}$ and $\nabla^{2}$ to $L$ coincide, and thus $\nabla_{B}^{1}=\nabla_{B}^{2}$.

In this approach the link between Magri's notion of compatibility and bi-affine compatibility follows immediately from Theorem 3 and is given in the following corollary.

Corollary 1. If the bi-Lagrangian foliation is a fibration with compact connected fibers, then Magri's compatibility implies bi-affine compatibility.

Proof of Theorem 3. (1) We construct the symplectic connections explicitly. The fact that $\nabla^{1}$ and $\nabla^{2}$ are symplectic and preserve $L$ will follow from the construction. Take an atlas of $M$ composed by Darboux-Nijenhuis charts. On each chart one can construct a torsion-free flat connection, symplectic with respect to $\omega_{1}$ and preserving $L$, by taking the linear connection whose Christoffel symbols vanish identically in these coordinates. One can then obtain a global connection by using partitions of unity to "glue" the connections obtained in each DarbouxNijenhuis chart. This construction gives the connection $\nabla^{1}$. For a more detailed explanation of this process see the proof of Theorem 2 in [7].

We now explain the construction of $\nabla^{2}$ in detail. Suppose we are in a Darboux-Nijenhuis chart. Let $\mathbf{z}=(\boldsymbol{\lambda}, \boldsymbol{\mu})$, then $\mathbf{e}_{i}=\frac{\partial}{\partial z^{i}}$ is a basis of tangent vectors. In these coordinates, the vanishing of the covariant derivative of $\omega_{2}$ is

$$
\left(\nabla_{\mathbf{e}_{k}}^{2} \omega_{2}\right)_{i j}=\frac{\partial}{\partial z^{k}}\left(\omega_{2}\right)_{i j}-\sum_{l} \Gamma_{i k}^{l}\left(\omega_{2}\right)_{l j}-\sum_{l} \Gamma_{j k}^{l}\left(\omega_{2}\right)_{i l}=0
$$

where the coefficients $\Gamma_{i j}^{k}$ are called Christoffel symbols. Since in Darboux-Nijenhuis coordinates we have

$$
\omega_{2}=\left[\begin{array}{cc}
\mathbf{0}_{n} & \boldsymbol{\Lambda}_{n}^{-1} \\
-\boldsymbol{\Lambda}_{n}^{-1} & \mathbf{0}_{n}
\end{array}\right]
$$

the term $\frac{\partial}{\partial z^{k}}\left(\omega_{2}\right)_{i j}$ is always zero except for

- $k=i, j=i+n$ with $1 \leq i \leq n$, in which case $\frac{\partial}{\partial \lambda i}\left(\omega_{2}\right)_{i(i+n)}=-\frac{1}{\lambda_{i}^{2}}$,

- $k=j, i=j+n$ with $1 \leq j \leq n$, in which case $\frac{\partial}{\partial \lambda j}\left(\omega_{2}\right)_{(j+n) j}=\frac{1}{\lambda_{j}^{2}}$.

Let us take all the $\Gamma_{i j}^{k}$ to be zero except the ones of the form $\Gamma_{i i}^{i}=-\frac{1}{\lambda_{i}}$ for $1 \leq i \leq n$. With this choice the Christoffel symbols are symmetric, and thus the connection is torsion-free.

To verify that $\nabla^{2}$ is symplectic with respect to $\omega_{2}$ we need to check only equation (3) for a few values of $i, j$ and $k$, since for all the other values the equation is trivially verified. We have

$$
\frac{\partial}{\partial z^{i}}\left(\omega_{2}\right)_{i(i+n)}-\Gamma_{i i}^{i}\left(\omega_{2}\right)_{i(i+n)}=\frac{\partial}{\partial \lambda_{i}}\left(\omega_{2}\right)_{i(i+n)}+\frac{1}{\lambda_{i}^{2}}=-\frac{1}{\lambda_{i}^{2}}+\frac{1}{\lambda_{i}^{2}}=0,
$$


where $1 \leq i \leq n$, and

$$
\frac{\partial}{\partial z^{j}}\left(\omega_{2}\right)_{(j+n) j}-\Gamma_{j j}^{j}\left(\omega_{2}\right)_{(j+n) j}=\frac{\partial}{\partial \lambda_{j}}\left(\omega_{2}\right)_{(j+n) j}-\frac{1}{\lambda_{j}^{2}}=\frac{1}{\lambda_{j}^{2}}-\frac{1}{\lambda_{j}^{2}}=0
$$

where $1 \leq j \leq n$. Therefore, the connection $\nabla^{2}$ is symplectic with respect to $\omega_{2}$.

To verify that $\nabla^{2}$ parallelizes $L$ we use the following coordinate formula

$$
\nabla_{\mathbf{e}_{j}}^{2} \mathbf{u}=\left(\frac{\partial u^{i}}{\partial z^{j}}+u^{k} \Gamma_{j k}^{i}\right) \mathbf{e}_{i}
$$

where, in general, $\mathbf{u}=\sum_{i} u^{i} \mathbf{e}_{i}$ is a vector field in $\Gamma(T M)$. Now suppose $\mathbf{u} \in \Gamma(L)$, then $\mathbf{u}=\sum i=n+1^{2 n} u^{i} \mathbf{e}_{i}$. Let $\mathbf{v}=\nabla_{\mathbf{e}_{j}}^{2} \mathbf{u}$. Since $\Gamma_{i j}^{k} \neq 0$ if and only if $i=j=k$, it follows that $\mathbf{v}$ is of the form

$$
\mathbf{v}=\sum_{i=n+1}^{2 n} a^{i} \mathbf{e}_{i}
$$

Since $u^{k} \neq 0$ only if $k \geq n+1$ and $\Gamma_{i j}^{k} \neq 0$ only when $1 \leq i \leq n$, then

$$
a^{i}= \begin{cases}\frac{\partial u^{i}}{\partial z^{j}}, & \text { if } i \geq n+1 \\ 0, & \text { in all other cases. }\end{cases}
$$

Thus, $\mathbf{v} \in \Gamma(L)$, that is, $\omega_{2}$ parallelizes $L$.

Now, taking an atlas covered with Darboux-Nijenhuis charts, passing to a locally finite refinement $\left(U_{\alpha}\right)_{\alpha \in A}$, denoting the corresponding family of linear connections constructed as above by $\left(\nabla_{\alpha}^{2}\right)_{\alpha \in A}$ and choosing a partition of unity $\left(\chi_{\alpha}\right)_{\alpha \in A}$ subordinate to the open covering $\left(U_{\alpha}\right)_{\alpha \in A}$, we can define

$$
\nabla^{2}=\sum_{\alpha \in A} \chi_{\alpha} \nabla_{\alpha}^{2}
$$

The conditions of parallelizing a given differential form, of parallelizing a given vector subbundle, and of being torsion-free are all local as well as affine. Thus, since each of the $\nabla_{\alpha}^{2}$ parallelizes $\omega_{2}$ and $L$, it follows that $\nabla^{2}$ also parallelizes $\omega_{2}$ and $L$.

(2) This part of the theorem follows immediately from Proposition 5.

(3) On each Darboux-Nijenhuis chart the restrictions of the connections $\nabla^{1}$ and $\nabla^{2}$ to the leaves of the foliation coincide, since for both connections the restriction of the Christoffel symbols will be of the form $\widetilde{\Gamma}_{i j}^{k}=\Gamma_{(i+n)(j+n)}^{k+n}$ with $1 \leq i, j, k \leq n$, and hence the Christoffel symbols $\widetilde{\Gamma}_{i j}^{k}$ of both connections vanish identically in these coordinates.

\section{Acknowledgments}

We would like to thank one of the anonymous reviewers for suggesting to us that Theorem 2 can be proved by using the uniqueness of the connection parallelizing all the Hamiltonian vector fields tangent to the leaves of a Lagrangian foliation. This work was supported by an NSERC Discovery Grant. 


\section{References}

[1] Bieliavsky P., Cahen M., Gutt S., Rawnsley J., Schwachhöfer L., Symplectic connections, Int. J. Geom. Methods Mod. Phys. 3 (2006), 375-420, math.SG/0511194.

[2] Bogoyavlenskij O.I., Theory of tensor invariants of integrable Hamiltonian systems. I. Incompatible Poisson structures, Comm. Math. Phys. 180 (1996), 529-586.

[3] Brouzet R., Systèmes bihamiltoniens et complète intégrabilité en dimension 4, C. R. Acad. Sci. Paris Sér. I Math. 311 (1990), 895-898.

[4] Falqui G., Pedroni M., Poisson pencils, algebraic integrability, and separation of variables, Regul. Chaotic Dyn. 16 (2011), 223-244.

[5] Fassò F., Ratiu T., Compatibility of symplectic structures adapted to noncommutatively integrable systems, J. Geom. Phys. 27 (1998), 199-220.

[6] Fernandes R.L., Completely integrable bi-Hamiltonian systems, J. Dynam. Differential Equations 6 (1994), 53-69.

[7] Forger M., Yepes S.Z., Lagrangian distributions and connections in multisymplectic and polysymplectic geometry, Differential Geom. Appl. 31 (2013), 775-807, arXiv:1202.5054.

[8] Gel'fand I.M., Dorfman I.Ja., Hamiltonian operators and algebraic structures related to them, Funct. Anal. Appl. 13 (1979), 248-262.

[9] Lee J.M., Introduction to smooth manifolds, Graduate Texts in Mathematics, Vol. 218, Springer-Verlag, New York, 2003.

[10] Magri F., A simple model of the integrable Hamiltonian equation, J. Math. Phys. 19 (1978), 1156-1162.

[11] Magri F., Casati P., Falqui G., Pedroni M., Eight lectures on integrable systems, in Integrability of Nonlinear Systems, Lecture Notes in Phys., Vol. 638, Editors Y. Kosmann-Schwarzbach, K.M. Tamizhmani, B. Grammaticos, Springer, Berlin, 2004, 209-250.

[12] Magri F., Morosi C., A geometrical characterization of integrable Hamiltonian systems through the theory of Poisson-Nijenhuis manifolds, Quaderni del Dipartimento di Matematica, Università di Milano, 1984.

[13] Olver P.J., Canonical forms and integrability of bi-Hamiltonian systems, Phys. Lett. A 148 (1990), $177-187$.

[14] Tondo G., Generalized Lenard chains and separation of variables, Quad. Mat. Univ. Trieste 573 (2006), $1-27$.

[15] Turiel F.-J., Classification locale simultanée de deux formes symplectiques compatibles, Manuscripta Math. 82 (1994), 349-362. 